\title{
Linking functional traits and network structure to concepts of stability
}

\author{
Z. Lindo \\ The University of Western Ontario, Department of Biology, London, Ontario, Canada N6A 5B7 \\ Email: zlindo@uwo.ca,phone: (1) 519-661-2111 ext 82284,fax: (1) 519-661-3935
}

Keywords: Diversity, Food webs, Functional traits, Network theory, Redundancy, Stability.

\begin{abstract}
The objectives of this paper are to discuss stability as an emergent property of ecological systems, as well as promote a common language of network theory that will transfer among socio-ecological systems. Many researchers have understood the relationship between diversity and redundancy of species as network nodes, and even the response/effect trait framework aspect of this (i.e., overlapping traits associated with environmental response and ecosystem-level effects). Many researchers have also made the connections between network architecture and robustness, recognizing species interaction links as important aspects of architecture, but few papers incorporate concepts of diversity and redundancy for both nodes and links into aspects of architecture, and consequently network dynamics and stability. Using a common language framework of network theory and commonalities of network properties across outwardly different network types may reveal the existence of common constraints or determinants on network stability for ecological systems.
\end{abstract}

\section{Introduction}

Growing anthropogenic impacts on ecological systems, concomitant with unprecedented rates of biodiversity loss have raised concern for the continued functioning of ecosystems and the services they provide. The capacity of an ecosystem to remain more or less in the same state, resist perturbation, or recover from disturbance is encapsulated in the 'meta-concept' (Loreau 2010) and multidimensionality (Donahue et al. 2013) of stability. Providing a definition for ecological stability is challenging (Mikkelson 1997) because the notion of stability changes depending on the ecological hierarchical level and spatio-temporal scale it is being measured at (Grimm and Wissel 1997, Loreau 2010). It is generally accepted that stability has three main components: the concept of defying change (resistance - a system that remains the same in spite of disturbance has a high resistance), the concept of rebounding from change (resilience - how quickly a system recovers from disturbance and returns to a steady state), and at the same time is often measured by a third factor - persistence or temporal stability (invariance - the ability of a system to maintain itself through time) (Loreau et al. 2002). In ecology we often use these components of stability concurrently to describe how an ecosystem will resist and recover from perturbation because ecologists are increasingly concerned with irreversible changes in natural systems. Much of what we describe is dictated by what we can measure, and thus there are many measures for these components of stability, such as measuring resistance through reactivity, persistence or robustness by the amount of perturbation that a system can tolerate before switching to another state, and the degree to which a variable changes after perturbation (for full review see Grimm and Wissel 1997). The problem with many of these metrics is that they are mostly dependent on measuring a response to disturbance - rather than being predictive of ecosystem stability. Having a predictive metric would confer benefits such as forecasting future system states, determine probable impacts, and facilitate options for mitigation or adaptation from a management perspective (Evans et al. 2013).

Understanding and predicting stability of biological systems is challenging because of this multidimensional nature, but also because the emergence of stability depends on the ecological hierarchical level and spatio-temporal scale it is measured at (Tilman 1996, Loreau 2010). Demonstrated factors affecting the stability of biological communities and ecosystems includes aspects of the biological community itself such as species diversity, trophic complexity and food web structure, and feedbacks among ecological hierarchical levels. In particular, a positive diversity-stability relationship is a long held notion in community ecology (e.g., MacArthur 1955), and despite arguments for and against the diversitystability hypothesis (e.g., Pimm and Lawton 1977), there is much experimental and observational support for increased biodiversity increasing both ecosystem stability and productivity (see Balvanera et al. 2006 for synthesis). From the very beginning MacArthur (1955) asserted that there is a stabilizing effect of diversity within biological systems due to increased number of the components (species) and their multiple interactions. Levin (1998) further suggested that this complexity (i.e., many parts, many types of interactions, and a dynamic nature of both leading to intractability) allows for and facilitates multiple feedbacks that lead to system stability. In this view of ecological systems as 'complex adaptive systems' (Levin 1998) it is recognized that both diversity and redundancy are integral parts of complexity, and that complexity leads to a dynamic system with feedbacks that allow 
Table 1. Network stability is increased through both node (species) and link (interaction) diversity and redundancy. Node diversity and redundancy pertains to both the response a node has to targeted loss (Response trait) as well as the ecological function that species performs (Effect trait) for stability to increase, stressing the importance of decoupling Response and Effect traits. Link diversity and redundancy increase network stability by creating an adaptive capacity for system flow (e.g., nutrients and energy).

\begin{tabular}{lll}
\hline & Description in ecological networks & $\begin{array}{l}\text { Associated species traits and } \\
\text { network properties }\end{array}$ \\
\hline Node diversity & Variability in response and effect traits of species & $\begin{array}{l}\text { Species richness; unique or specialist species; ver- } \\
\text { tical trophic diversity }\end{array}$ \\
Node redundancy & Multiplicity of response and effect species traits & $\begin{array}{l}\text { Compensatory dynamics; generalist species; de- } \\
\text { coupling of response and effect traits; horizontal } \\
\text { trophic diversity }\end{array}$ \\
Link diversity & Variability of interaction types & $\begin{array}{l}\text { Interaction directionality, interaction strength, mu- } \\
\text { tualisms and antagonisms }\end{array}$ \\
Link redundancy & $\begin{array}{l}\text { Presence of multiple and/or alternative interac- } \\
\text { tions among nodes }\end{array}$ & $\begin{array}{l}\text { Generalist species, high link density, indirect ef- } \\
\text { fects, hub and connector species }\end{array}$ \\
\hline
\end{tabular}

for adaptive responses. Further discussions of ecological complex adaptive systems in the context of anthropogenic change (Levin et al. 2013) suggest that by understanding the emergent properties of complex systems we can alter how we interact with natural systems to prevent catastrophic regime shifts, by identifying and pulling back from tipping points (Levin and Lubchenco 2008).

Placing the idea of stability within a common language framework, I utilize network theory, which has developed from graph theory in the computer and math sciences. Network theory describes the relationships (links) among discrete objects (nodes). In ecological networks, nodes are species, and links are antagonistic or mutualistic interactions. The application of network theory has already been useful to various disciplines including social and ecological theories (Montoya et al. 2006). In particular, ecological networks are now heavily used to represent food webs and other interaction (e.g., mutualistic) webs (Ings et al. 2009, Thébault and Fontaine 2010, Bascompte 2007, 2009a). As biological interaction descriptors, ecological networks can be used to describe and compare the structure of communities (Dunne et al. 2002), while theoretical network models can be used to investigate the effects of this structure on higher order properties such as ecosystem stability (Allesina and Pascual 2008, Okuyama and Holland 2008). By removing the specific context of the system (e.g., network versus food web), network theory can unify multiple concepts of stability to better understand the patterns and processes underlying it.

\section{Stability arises from diversity and redundancy}

In this section I outline how stability, as a function of a complex adaptive system, can be described through network theory principles. Network theory has two components as described above (nodes and links); each of these components has two factors that lead to complexity (diversity (i.e., variability) and redundancy (i.e., multiplicity)), which in combination, lead to the adaptiveness of the system as a whole. MacArthur (1955) proposed that the more diverse and interconnected a community is, the more resilient it will be in the face of disturbance. Here I expand on this to suggest that, while MacArthur invoked the concept of nodes (MacArthur's 'diversity') and links (MacArthur's 'interconnectedness'), these two elements both need to contain diversity and redundancy to create stability. In this way, diversity and redundancy form complementary aspects (or axes) of complexity; both nodes and links of a network require diversity and redundancy for the network to be complex and adaptive (Table 1).

\subsection{Diversity and redundancy of nodes}

If we place the concept of diversity and redundancy of nodes within the familiar context of an ecological food web, we can consider each node as a different species or feeding group, and the links among species as feeding relationships. In this case, a species must interact trophically to be part of the web, thus more species in the web leads to more overall feeding interactions. A greater number of species in the system automatically increase the complexity of the system first, by directly having more species (nodes), and second, by increasing the minimum number of interactions (links) that are required for all species to be considered part of the web. Even if we consider a community of species at a single trophic level, interactions such as competition link the components of the community. Here, how species diversity leads to temporal stability is described in ecology through the concept of the portfolio effect (Doak et al. 1998, Tilman et al. 1998), which is analogous to a stock holding or investment portfolio, where increased diversity within the portfolio minimizes the instability of the overall 'investment'. The portfolio effect of node diversity can arise from two mechanisms: statistical averaging or a niche-based effect of compensatory dynamics. Through statistical averaging, the total variance of a collection of random variables is lower when there are more variables (Lehman and Tilman 2000). Compensatory dynamics are niche-based in that they assume that competing species will negatively covary; some species do well because other species are performing poorly. This asynchrony may arise due to dissimilarities in how species respond to environmental fluctuations, or how fast species respond and recover from perturbations (Loreau and de Mazencourt 2013). The strength of the portfolio effect is not just reliant on the number of spe- 
cies, but also the evenness of abundances (Doak et al. 1998, Thibaut and Connolly 2013), which can increase or decrease the effect of the portfolio effect depending on dynamics of the individual species included (Thibaut and Connolly 2013). Both statistical averaging and compensatory dynamics depend on species having independent response dynamics to external perturbations, which assumes that different species respond to perturbation differently, while performing similar ecosystem functions, i.e., functional redundancy. Both these mechanisms invoke functional complementarity through compensatory dynamics of nodes, whether at random, or due to competitive negative covariance, based on differential traits in how species respond to change.

This leads into a current discussion in biodiversityecosystem function research of response and effect traits (Lavorel and Garnier 2002). In short, response traits describe how a species responds to environmental conditions and which are affected by change, while effect traits are those that relate to ecosystem-level processes (Lavorel and Garnier 2002, Suding et al. 2008). If all species have a differential response to perturbation, then the averaging of these responses will be stable over time. Yet, if having more different types of species has an averaging effect on the overall system, complexity in the context of stability is still only increased along one potential axis. The second axis depends on redundancy among those nodes - having multiples of nodes that perform the same function. This redundancy to a certain extent is related to diversity, as increasing the number of nodes increases the likelihood of having redundant nodes. Here, the random loss of a node performing a given function is compensated by other nodes doing the same thing, but only for random (stochastic) losses, which are independent from how a node responds to a given perturbation, and the functional effect that node has on the system.

If functionally nodes respond the same way and affect the same ecosystem-level process (i.e., response and effect traits are the same), then any non-random loss could eliminate all nodes that perform a specific function, and ecosystem stability is decreased (Figure 1A). But, if a system has a greater number of nodes that either respond differently to perturbation and/or perform different functions, then there is coverage through redundancy (Ives and Carpenter 2007). This is referred to as the insurance hypothesis (Yachi and Loreau 1999). For instance, if an ecosystem has a greater number of species, it increases the likelihood that either species that respond to a given perturbation will perform different functions, or that species that perform the same function will respond differently to the same perturbation. This hypothesis necessitates the decoupling of response and effect traits. So the axis of redundancy that leads to complexity and stability must be at two levels; how nodes respond (response traits) and the function that a node performs (effect traits), and the decoupling of response and effect traits. Taken together, it stands that trait differences are the underlying cause of diversity-stability relationships, functional redundancy within functional groups is critical for stability (Fonseca and Ganade 2001), and the diversity of response traits is likely more important than the redundancy of response traits (Elmqvist et al.
2003, Mori et al. 2013) (Figure 1B). Suding et al. (2008) outline the possible outcomes of different relationships between response and effect traits, and present several empirical cases where response traits correlate with effect traits (e.g., Larsen et al. 2005, Solan et al. 2004, Engelhardt 2006) leading to significant changes in ecosystem function. Yet understanding which traits are important and explicitly linking response and effect traits is expected to remain problematic (Lavorel and Garnier 2002).

\subsection{Diversity and redundancy of links}

Similar to how diversity and redundancy of nodes can lead to stability through averaging and insurance effects, diversity and redundancy of network links is hypothesized to increase the stability of networks (Figure 1C). Link diversity can be enhanced through three areas of differentiation; links can differ in the direction and type of link directionality (unidirectional or bidirectional), strength of interaction (e.g., weak to strong, indirect vs. direct), or the type of interaction itself (antagonistic or mutualistic). Different types of interactions among species can be antagonistic, including feeding or trophic interactions, competition interactions, or parasitism, while beneficial or facultative interactions can be mutualisms and commensalisms; combinations of these elements leads to interactions being symmetrical (e.g., $(+/+)(-/-))$ or asymmetrical (e.g., (+/-) (+/0) (-/0)). While the recognition and historical focus on food webs in ecology has led to many theories and metrics of network structure now being testable, there has been an over-representation of this single type of interaction network; food webs are a collection of typically unilateral, asymmetrical, direct antagonistic interactions. As such, our understanding of multi-categorical networks is still in infancy as we begin to recognize and undertake studies of other interaction paradigms (e.g., mutualistic networks) (but see Bascompte 2009a).

Link redundancy is harder to define than link diversity. Whether link redundancy is a by-product of node redundancy or whether combinations or different configurations of links increase link redundancy is unknown. One thought is that generalist feeders and nodes with high link density increase redundancy of links; i.e., route redundancy (sensu Rayfield et al. 2011) which considers the presence of multiple and alternative pathways among nodes including indirect effects. Tylianakis et al. (2010) review the structural attributes of ecological networks, both mutualistic and antagonistic, to discuss the within system and emergent features that confer stability. They repeatedly identify a complementary relationship between interaction diversity and redundancy. For instance, basic measures of link diversity among nodes (linkage density (average links per node), or measures of connectance (e.g., links/nodes 2$)$ ) can increase ecosystem function and the stability of function through increased link redundancy (Tylianakis et al. 2010). Thus, stability of function arises through multiplicity of pathways towards an end point, similar to traffic rerouting in a construction zone; there need to be similar, but alternative routes that will continue to flow to the 


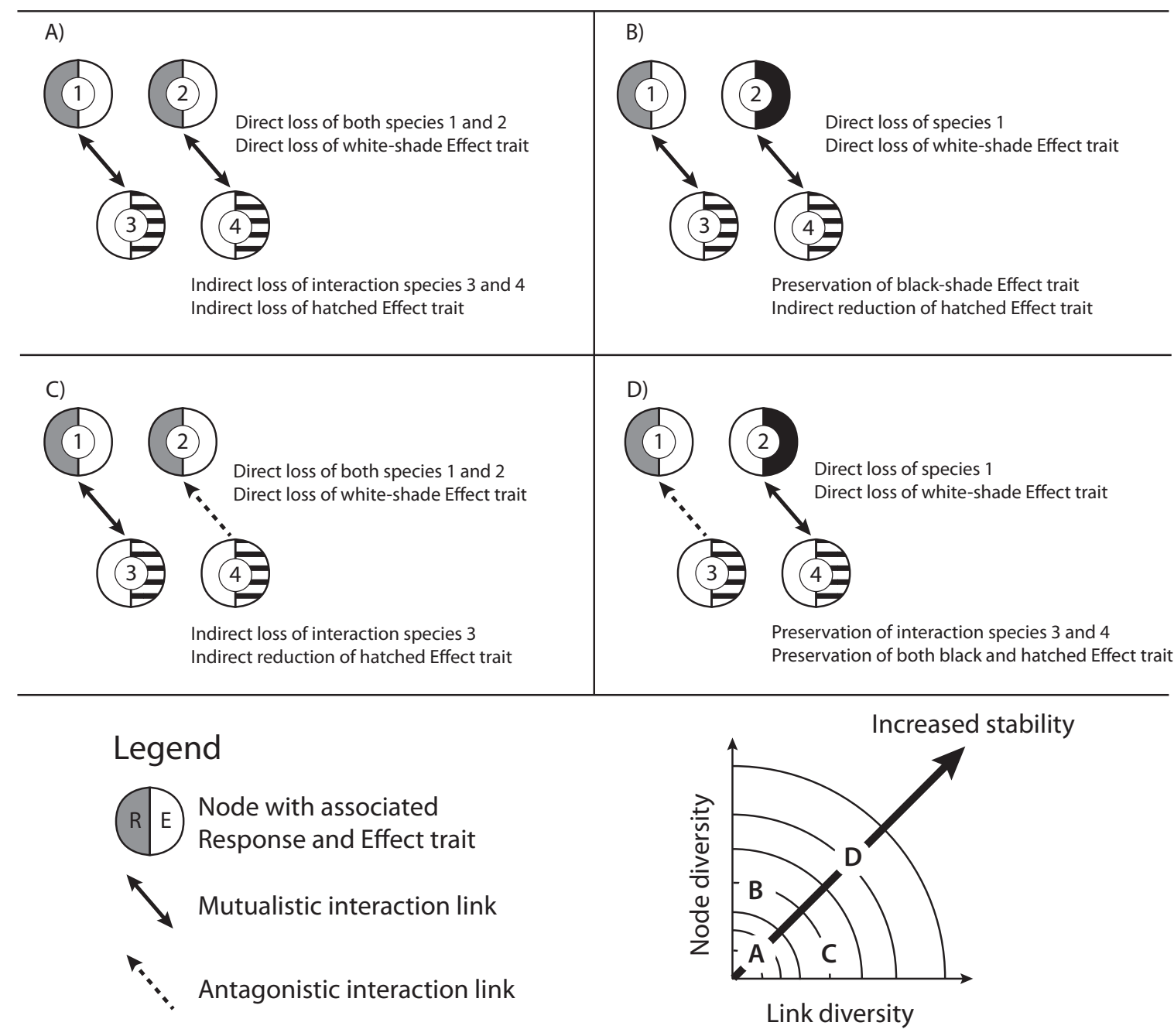

Figure 1. Conceptual diagram of two species interaction scenarios under targeted loss of species with grey-shade Response trait. Each species is shown as a circular node with an associated Response (left) and Effect (right) trait. Species 1 and 2 interact with species 3 and 4 respectively, in either a mutualistic or antagonistic interaction link. A) Low node and low link diversity result in low stability of the network at both species and ecosystem functional scales; B) higher node diversity and low link diversity; C) higher link diversity and low node diversity; D) higher node and higher link diversity demonstrate increased stability at both species and ecosystem functional scales. Scenarios shown are four simple examples of a possible 576 permutations in this system; more complex combinations of node and link diversity are expected to further increase stability.

same destination. Therefore it appears that link diversity and link redundancy are tightly coupled.

Similarly the variability of link strengths among nodes has implications for overall network structure, and by extension the stability of the network (Yeakel et al. 2012). Differential distribution of link strength (many weak, few strong) has been shown in many ecological networks in recent years, and has led to an understanding that weak interactions are stabilizing, but dependent on the arrangement of interactions (McCann et al. 1998). Mougi and Kondoh (2012) found that the presence of only a few weak links provided no stability; rather it is the interaction of node and link diversity that leads to stability. They refer to this as a 'hybrid' community type and demonstrate that the relationship between diversity and stability depends on the type of diversity - increased along just one axis is destabilizing (e.g., high node richness and strong interactions), versus when diversity is increased along both node and link axes (Figure 1D). This work highlights the connection between the diversity of both nodes and links; increased node richness increases the absolute number of links, yet the opposite is not necessarily true, thus measures of link density can indicate robust networks (Dunne et al. 2002). In this way, increased node richness can alter mean link strength and stability - the 'weak-interaction effect' - suggesting that interactions will be on average weaker in a more diverse community, and therefore more stable (McCann 2000). With this, direct interactions are thought to be stronger than indirect interactions (relationships through intermediary nodes), and the effects of which, the latter are expected to take longer to emerge (Schoener 1993) and dampen cascading effects through networks (e.g., secondary extinctions). 
Interaction type, whether antagonistic as in trophic webs, or beneficial as in mutualistic webs will also dictate strength and directionality of links. Competitive networks are poorly studied, but competition among species can lead to compensatory dynamics, correspondingly increased resource use, and so-called 'hypercycles', which are positive feedback, three species loops reminiscent of the game 'rock, paper, scissors', that can increase biodiversity (e.g., Lankau and Strauss 2007). Mutualistic networks also contain hypercycles and other distinct network architecture that confers stability (Bascompte 2009a); mutualisms are generally weaker in link strength than antagonisms, mutualistic networks are nested and asymmetrical. That is, specialist species interact with a subset of the species that generalists interact with; core species are generalists, create redundant links, and have shorter paths between any two species versus food webs (Bascompte et al. 2003). It is this highly connected and nested architecture that promotes community stability in mutualistic networks, whereas stability is increased in weakly connected and compartmentalized antagonistic networks as described below.

\section{Network structure emerges from node and link di- versity and redundancy}

We can describe interaction webs by numerous metrics including the number of nodes (i.e., species richness), the number of links between the nodes (i.e., species interactions), or by the nature of the links (i.e., antagonistic or mutualistic), and their strengths (Bascompte 2009b). General properties are being described through the concept of 'motifs', which are unique link patterns that incorporate all different link elements (e.g., uni- and bidirectional, antagonistic and synergistic elements). The importance of network topology and the presence of stabilizing motifs are an area of current research (Milo et al. 2002, Rayfield et al. 2011). One difference between mutualistic and antagonistic networks is mutualistic networks are often strongly nested, while antagonistic networks are more modular/compartmentalized (Yeakel et al. 2012). Rooney and McCann (2012) highlight the importance of modularity in comparing 'slow' (detrital) versus 'fast' (production) subwebs of a marine food web. They demonstrate that the mean number of links (link density) is greater in the slow food web channel indicating more weak interactions and greater link redundancy than the fast channel, but it was the coupling of the two subwebs (fast and slow) that lead to higher stability. Gonzalès and Parrott (2012) show that compartmentalism, which creates subwebs (modularity), is important for controlling the flow of interactions among nodes where certain nodes act as brokers, but there is a balance between modularity and subgroup connectance that leads to robustness; intermediate modularity is the key. The species that connect different subwebs (i.e., connectors) need not be wellconnected (i.e., hubs), but play an important role in the overall network structure. A balance between connectance and modularity suggests that the system will be robust to random node removal (Anand et al. 2010), but targeted loss of hubs has the potential to lead to collapse.
In the case of food webs, mid-trophic level species connect basal trophic positions with predators, as well as often linking several subwebs (Lai et al. 2012). Under perturbation, extinction risk is typically non-random and greater in higher trophic-level species, or other species with larger body size, and smaller local population densities (Didham et al. 1998, Cardillo 2003). While top predators are not typically hub species, they can integrate several food webs over space (Pillai et al. 2011), and the loss of predators can have cascading effects that manifest at the ecosystem-level (Staddon et al. 2010). Lai et al. (2012) also show that unique species were vulnerable to perturbation while mid-trophic-level species were less vulnerable, while at the same time dampening cascading effects to other nodes in the network (Livi et al. 2011).

\section{What does network theory mean for stability?}

The emergent properties of network structure as described above are important for how perturbation to the system can propagate through the system, where even the loss of indirect interactions are shown to have cascading effects (Rodriguez-Cabal et al. 2013). But at the same time, these diversity and redundancy elements of nodes and links lead to an adaptiveness as well as the complexity of the system. Ecologist's perception of complexity revolves around diversity, heterogeneity, interactions or connectivity, and historic or memory-based effects. Networks with high diversity and redundancy of links provide feedback loops and legacy effects that include a temporal disconnected from the original effect through indirect interactions, and a memory of past events. In ecological networks this is seen through delayed effects of cascading secondary extinctions during the process of community disassembly following perturbation and what is now referred to as the extinction debt (Gonzalez 2000), and through co-evolutionary relationships particularly in mutualistic networks (Nuismer et al. 2013). Connectivity, when strong can increase the likelihood of spreading or scaling-up of local instability to larger scales. However connectivity is essential for the adaptiveness of the system (Hughes et al. 2013).

Using a common language framework of network theory and commonalities of network properties across outwardly different network types may reveal the existence of common constraints or determinants on network stability for ecological systems. For instance, Thébault and Fontaine (2010) show that the effect of connectance (linkages) and diversity (node richness) on measures of stability (persistence and resilience) in both mutualistic and trophic networks is mostly modulated through aspects of network topology (modularity, nestedness). The importance of connectance within subwebs combined with hub nodes creating modularity suggests network robustness is built through link redundancy (Salles and Marino 2012), but highlights the idea that there may be tradeoffs among diversity and redundancy along certain axes of stability. For nodes, diversity of response and effect traits confers stability through the portfolio effect and multifunctionality, but is only truly realized when there are also redundancy in effect traits, and response and effect traits are 
decoupled (insurance hypothesis). Here while the emergent property (ecosystem level functionality) is stabilized at the expense of population variability (Tilman 1996), the overall stability of the network (community level) will ultimately depend on the diversity and redundancy of the interactions links. It is here, at this intermediate ecological hierarchy of the community that metrics or indicators of stability may provide novel insight into thresholds of natural systems. This will require the parameterization of real ecological networks that encapsulate both node diversity and redundancy as historically attempted, but also the diversity and redundancy of interactions among species.

Acknowledgements. I would like to thank two anonymous reviewers and F. Jordán for valuable comments and suggestions on this manuscript. Thanks to E. Desjardin, G. Barker, A. Dussault (Rotman Institute, Dept. Philosophy, University of Western Ontario) and C. Dieleman (Dept. Biology, University of Western Ontario) for lively discussions on resilience paradigms in ecology and philosophy. I acknowledge funding from the Natural Sciences and Engineering Research Council of Canada.

\section{References}

Allesina, S. and Pascual, M. 2008. Network structure, predator-prey motifs, and stability in large food webs. Theor. Ecol. 1: 55-64.

Anand, M., Gonzalez, A., Guichard, F., Kolasa, J. and Parrott, L. 2010. Ecological systems as complex systems: challenges for an emerging science. Diversity 2: 395-410.

Balvanera, P., Pfisterer, A.B., Buchmann, N., He, J.S., Nakashizuka, T., Raffaelli, D. and Schmid, B. 2006. Quantifying the evidence for biodiversity effects on ecosystem functioning and services. Ecol. Lett. 9: 1146-1156.

Bascompte, J. 2007. Networks in ecology. Basic Appl. Ecol. 8: 485 490.

Bascompte, J. 2009a. Mutualistic Networks. Front. Ecol. Envir. 8: 429-436.

Bascompte, J. 2009b. Disentangling the web of life. Science 325 416-419.

Bascompte, J., Jordano, P., Melián, C.J. and Olesen, J.M. 2003. The nested assembly of plant-animal mutualistic networks. PNAS 100: 9383-9387.

Cardillo, M. 2003. Biological determinants of extinction risk: why are smaller species less vulnerable? Anim. Conserv. 6: 63-69.

Didham, R.K, Hammond, P.M., Lawton, J.H., Eggleton, P. and Stork, N.E. 1998. Beetle species responses to tropical forest fragmentation. Ecol. Monogr. 68: 295-323.

Doak, D.F., Bigger, D., Harding, E.K., Marvier, M.A., O’Malley, R.E. and Thomson, D. 1998. The statistical inevitability of stability-diversity relationships in community ecology. Am. Nat. 151: $264-276$.

Donahue, I., Petchey, O.L., Montoya, J.M., Jackson, A.L., McNally, L., Viana, M., Healy, K., Lurgi, M., O’Connor, N. and Emmerson, M. 2013. On the dimensionality of ecological stability. Ecol. Lett. 16: 421-429.

Dunne, J.A., Williams, R.J. and Martinez, N.D. 2002. Network structure and biodiversity loss in food webs: robustness increases with connectance. Ecol. Lett. 5: 558-567.
Elmqvist, T., Folke, C., Nyström, M., Peterson, G., Bengtsson, J., Walker, B. and Norberg, J. 2003. Response diversity, ecosystem change, and resilience. Front. Ecol. Envir. 1: 488-494.

Engelhardt, K. 2006. Relating effect and response traits in submersed aquatic macrophytes. Ecol. Appl. 16: 1808-1820.

Evans, M.R., Bithell, M., Cornell, S.J., Dall, S.R., Díaz, S., Emmott, S., Ernande, B., Grimm, V., Hodgson, D.J., Lewis, S.L., Mace, G.M., Morecroft, M., Moustakas, A., Murphy, E., Newbold, T., Norris, K.J., Petchey, O., Smith, M., Travis, J.M. and Benton, T.G. 2013. Predictive systems ecology. Proc. Roy. Soc. B. 280: 20131452.

Fonseca, C. and Ganade, G. 2001. Species functional redundancy, random extinctions and the stability of ecosystems. J. Ecol. 89: 118-125.

Gonzalès, R. and Parrott, L. 2012. Network theory in the assessment of the sustainability of social-ecological systems. Geography Compass 6: 76-88.

Gonzalez, A. 2000. Community relaxation in fragmented landscapes: the relation between species richness, area and age. Ecol. Lett. 3: 441-448.

Grimm, V. and Wissel, C. 1997. Babel, or the ecological stability discussions: an inventory and analysis of terminology and a guide for avoiding confusion. Oecologia 109: 323-334.

Hughes, T.P., Carpenter, S., Rockström, J., Scheffer, M. and Walker, B. 2013. Multiscale regime shifts and planetary boundaries. Trend Ecol. Evol. 28: 389-395.

Ings, T.C., Montoya, J.M., Bascompte, J., Blüthgen, N., Brown, L., Dormann, C.F., Edwards, F., Figueroa, D., Jacob, U., Jones, J.I., Lauridsen, R.B., Ledger, M.E., Lewis, H.M., Olesen, J.M., van Veen, F.J., Warren, P.H. and Woodward, G. 2009. Ecological networks - beyond food webs. J. Animal Ecol. 78: 253-269.

Ives, A.R. and Carpenter, S.R. 2007. Stability and diversity of ecosystems. Science 317: 58-62.

Lai, S.-M., Liu, W.-C. and Jordán, F. 2012. On the centrality and uniqueness of species from the network perspective. Biol. Lett. 8: 570-573.

Lankau, R.A. and Strauss, S.Y. 2007. Mutual feedbacks maintain both genetic and species diversity in a plant community. Science 317: 1561-1563

Larsen, T.H., Williams, N.M. and Kremen, C. 2005. Extinction order and altered community structure rapidly disrupt ecosystem functioning. Ecol. Lett. 8: 538-547.

Lavorel, S. and Garnier, E. 2002. Predicting changes in community composition and ecosystem functioning from plant traits: revisiting the Holy Grail. Funct. Ecol. 16: 545-556.

Lehman, C.L. and Tilman, D. 2000. Biodiversity, stability, and productivity in competitive communities. Am. Nat. 156: 534-532.

Levin, S.A. 1998. Ecosystems and the biosphere as complex adaptive systems. Ecosystems 1: 431-436.

Levin, S.A. and Lubchenco, J. 2008. Resilience, robustness, and marine ecosystem-based management. BioScience 58: 27-32.

Levin, S.A., Xepapadeas, T., Crépin, A.-S., Norberg, J., de Zeeuw, A., Folke, C., Hughes, T., Arrow, K., Barrett, S., Daily, G., Ehrlich, P., Kautsky, N., Mäler, K.-G., Polasky, S., Troell, M., Vincent, J.R. and Walker, B. 2013. Social-economic systems as complex adaptive systems: Modeling and policy implications. Environ. Develop. Econom. 18: 111-132.

Livi, C.M., Jordán, F., Lecca, P. and Okey, T.A. 2011. Identifying key species in ecosystems with stochastic sensitivity analysis. Ecol. Model. 222: 2542-2551.

Loreau, M. 2010. Linking biodiversity and ecosystems: towards a unifying ecological theory. Phil. Trans. Roy. Soc. B 365: 49-60. 
Loreau, M. and de Mazencourt, C. 2013. Biodiversity and ecosystem stability: a synthesis of underlying mechanisms. Ecol. Lett. 16: 106-115.

Loreau, M., Downing, A., Emmerson, M., Gonzalez, A., Hughes, J., Inchausti, P., Joshi, J., Norberg, J. and Sala, O. 2002. A new look at the relationship between diversity and stability. In: Loreau, M., Naeem, S. and Inchausti, P. (eds.), Biodiversity and Ecosystem Functioning: Synthesis and Perspectives. Oxford University Press. pp. 79-91.

MacArthur, R.H. 1955. Fluctuations of animal populations and a measure of community stability. Ecology 36: 533-535.

McCann, K.S. 2000. The diversity-stability debate. Nature 405: 228233.

McCann, K., Hastings, A. and Huxel, G.R. 1998. Weak trophic interactions and the balance of nature. Nature 395: 794-798.

Mikkelson, G.M. 1997. Methods and metaphors in community ecology: the problem of defining stability. Perspectives on Science 5: 481-498.

Milo, R., Shen-Orr, S., Itzkovitz, S., Kashtan, N., Chklovskii, D. and Alon, U. 2002. Network motifs: simple building blocks of complex networks. Science 298: 824-827.

Montoya, J.M., Pimm, S.L. and Sole, V.R. 2006. Ecological networks and their fragility. Nature 442: 259-264.

Mori, A.S., Furukawa, T. and Sasaki, T. 2013. Response diversity determines the resilience of ecosystems to environmental change. Biol. Rev. 88: 349-364.

Mougi, A. and Kondoh, M. 2012. Diversity of interaction types and ecological community stability. Science 337: 349-351.

Nuismer, S.L., Jordano, P. and Bascompte, J. 2013. Coevolution and the architecture of mutualistic networks. Evolution 67: 338-354.

Okuyama, T. and Holland, J.N. 2008. Network structural properties mediate the stability of mutualistic communities. Ecol. Lett. 11: 208-216.

Pillai, P., Gonzalez, A. and Loreau, M. 2011. Metacommunity theory explains the emergence of food web complexity. PNAS 108: 19293-19298.

Pimm, S.L. and Lawton, J.H. 1977. The number of trophic levels in ecological communities. Nature 268: 329-331.

Rayfield, B., Fortin, M.-J. and Fall, A. 2011. Connectivity for conservation: a framework to classify network measures. Ecology 92: 847-858.

Rodriguez-Cabal, M.A., Barrios-Garcia, M.N., Amico, G.C., Aizen, M.A. and Sanders, N.J. 2013. Node-by-node disassembly of a mutualistic network driven by species introductions. PNAS 110: 16503-16507.
Rooney, N. and McCann, K.S. 2012. Integrating food web diversity, structure and stability. Trends Ecol. Evol. 27: 40-46.

Salles, R.M. and Marino, D.A., Jr. 2012. Strategies and metrics for resilience in computer networks. The Computer Journal 55: 728739.

Schoener, T.W. 1993. On the relative importance of direct versus indirect effects in ecological communities. In: Kawanabe, H., Cohen, J.E. and Iwasaki, K. (eds.), Mutualism and Community Organization. Oxford University Press. pp. 365-411.

Solan, M., Cardinale, B.J., Downing, A.L., Engelhardt, K.A.M., Ruesink, J.L. and Srivastava, D.S. 2004. Extinction and ecosystem function in the marine benthos. Science 306: 1177-1180.

Staddon, P., Lindo, Z., Crittenden, P.D., Gilbert, F. and Gonzalez, A. 2010. Connectivity, non-random extinction, and ecosystem function in experimental metacommunities. Ecol. Lett. 13: 543552.

Suding, K.N., Lavorel, S., Chapin III, F.S., Cornelissen, J.H.C., Díaz, S., Garnier, E., Goldberg, D., Hooper, D.U., Jackson, S.T. and Nava, M.-L. 2008. Scaling environmental change through the community-level: a trait-based response-and-effect framework for plants. Glob. Change Biol. 14: 1125-1140

Thébault, E. and Fontaine, C. 2010. Stability of ecological communities and the architecture of mutualistic and trophic networks. Science 329: 853-856.

Thibaut, L.M. and Connolly, S.R. 2013. Understanding diversity-stability relationships: towards a unified model of portfolio effects. Ecol. Lett. 16: 140-150.

Tilman, D. 1996. Biodiversity: population versus ecosystem stability. Ecology 77: 350-363.

Tilman, D., Lehman, C.L. and Bristow, C.E. 1998. Diversity-stability relationships: statistical inevitability or ecological consequence? Am. Nat. 151: 277-282.

Tylianakis, J.M., Laliberté, E., Nielsen, A. and Bascompte, J. 2010. Conservation of species interaction networks. Biol. Conserv. 143: 2270-2279.

Yachi, S. and Loreau, M. 1999. Biodiversity and ecosystem productivity in a fluctuating environment: the insurance hypothesis. PNAS 96: 1463-1468.

Yeakel, J.D., Guimaraes, P.R., Novak, M., Fox-Dobbs, K. and Koch, P.L. 2012. Probabilistic patterns of interaction: the effects of link-strength variability on food web structure. J. Roy. Soc. Interface 9: 3219-3228.

Received April 8, 2014 Revised June 24, August 8, 2014 Accepted September 6, 2014 